\title{
Down's syndrome and Alzheimer Disease Imaging Genetics
}

\author{
Ahmed Bashir MD, $\mathrm{PhD}^{1 *}$ and Stanley Nelson $\mathrm{MD}^{2}$ \\ ${ }^{1}$ Department of Pediatrics, Al Mana General Hospital, Jubail, P.O. BOX10366, 113 Al Hawailat Street, K.S.A, 31961 \\ ${ }^{2}$ Professor of Genetics, David Geffen Medical School, UCLA,CA, USA
}

*Corresponding author: Ahmed Bashir, Department of Pediatrics, Al Mana General

Received Date: December 17, 2018

Hospital, Jubail, P.O. BOX10366, 113 Al Hawailat Street, K.S.A, 31961.

Published Date: January 24, 2019

\section{Mini Review}

The advancement of both in vivo imaging modalities that detect the neuropathologist associated with both Down's syndrome and Alzheimer's disease present new opportunities to explore these diseases in living human subjects. Previously, these neuropathologists could not be detected until after autopsy or in the living patient, with the rarely taken brain biopsy. The use of quantitative traits derived from these imaging modalities offers increased power to detect associations with large scale genetic data, and these studies fall under the category of imaging genetics.

Imaging genetics studies can identify novel risk genes and elucidate gene function and novel mechanisms disease pathology and etiology. Recent imaging genetics studies of the neuropathologist of Ad and DS Have attempts to obtain a more complete and in depth understanding the underlying genetic etiology of their pathogeneses. Here we will briefly overview both diseases, the neuropathologist associated with each, the imaging modalities used to detect these pathologies, and finally, the studies combing these imaging modalities with genetic data.

Utilizing imaging modalities in genetic studies by leveraging quantitative traits drive from imaging modalities, researches can take advantage of the increase power this trait provide genetic association studies [1]. In order to do so, one must define the trait of first, to define phenotype or disease status in genetic studies as in genetic clinic physicians and scientist traditionally depend on a patient clinical symptom as measured through a battery of cognitive test [2]. They are strength and weaknesses to using a solely clinical approach in defining outcome variables in genetic studies. This approach matches the diagnosis made in clinical settings and this cognitive measure have been validated, normalize, and in use for the decades, which allows data to be complied across research centers.

Despite this advantage the gold standard for diagnosis of $\mathrm{AD}$ at autopsy, the diagnostic accuracy of new cognitive test ranges from 65 to $96 \%$ and the specificity range between 23 to $28 \%$
45. Therefore, using only clinical diseases status, as diagnose by cognitive and neuropsychological test, in genetic association status studies introduces potential error into the result. They are many effects of genetic variant - from protein to cell function to system physiology - that are intermediate to final disease status. Measuring these intermediate effects can provide endophenotypes for a genetic association study, potentially increasing the power to detect a genetic effect that ultimately impacts disease status [3]. To qualify this intermediate effect, biomarkers like protein level in cerebrospinal fluid (CSF) or quantitative neuroimaging modalities like MRI and PET, have been added to research criteria for ad diagnosis 3 and have been used the genetic studies. For AD, DS, other neurological orders brain structure or pathology drive from imaging modalities can be the source of relevant quantitative test (QTs) to be used as endophenotypes. Endophenotype can provide increase statistical power (and therefore decrease sample size requirements) over dichotomous outcome variables [4]. And since both brain structure [5]. And PET- drive amyloidal load 6. Are highly heritable, both are suitable as endophenotypes in genetic studies.

In recognition of this great opportunity and the need of large sample sizes for exploration of modest genetics effects, the AD neuroimaging initiative (ADNI) was developed. ADNI as a joint ventures between the national institutive health $(\mathrm{NIH})$ and private biotechnology and pharmaceutical companies with the goal of acquiring serial measurements of MRI, PET, neurocognitive testing and other biological markers, along with genetic data in hundreds of adults for the purpose of identifying biomarkers for early detection of monitoring of disease progression and response to treatment for AD. ADNI maintains a publicly available dataset that contains reach biomarker data on study participants with normal cognitive and those diagnose MCI or AD. This is an extra ordinary resource for $\mathrm{AD}$ researchers, especially those interested in imaging genetic research. The availability of ADNI and other similarly size data sets has advance it the field of imaging genetics in LOAD, by enabling the use of quantitative structural data from MRI and quantities 
amyloidal load from PET as endophenotype in genetic association studies.

Structural MRI and quantified PET images are able to successfully quantify different aspects of brain pathology and these quantitative traits have been used in genetic association's studies to confirm previous and identify novel genetic association.

Although researchers have not yet found a specific gene that is determinative of the late onset of $\mathrm{AD}$, one genetic risk factor. APOE is located on chromosome 19 and encodes the protein Apo lipoprotein $\mathrm{E}$ that combines with lipids to form lipoproteins. They are 3 variant of this gene, APOE e2, APOE e3, and APOE e4 (average frequency in percentage: $6,4,78.3,14.5$, respectively) [6]. APOE e3 is the most common allele and, therefore risk attributed to other allelic variants is compared against this reference allele. APOE e2 may provide some protection against the disease in younger individuals [7]. Risk for AD is highest in individuals who possess an APOE e4 allele. An individual's risk for developing AD increase from $20 \%$ with no APOE e 4 alleles are present to $90 \%$ when 2 copies of the allele are present [8]. It is important to know; however, that the increase rick conferred by APOE e4 is lower for African Americans and Hispanics than it is for Caucasians [9].

Using AV-45 ligand PET images and genetic data collected by ADNI on 555 subjects, Ramanan, et.al, confirmed the association of APOE (rs 429358) with amyloid load in possession patient subjects [10]. In facts there is a gene dosage effect of the e4 risk allele on PET-derived amyloid load that has been observed in cognitively normal people 11 as well as MCI subjects [11]. APOE e4 allele has also been associated with faster conversion rates from MCI to AD in PIB- positive MCI subjects [12]. In genome wide association study (GWAS) using quantitative endophenotypes drive from MRI, APOE has also be associated with multiple measures of brain volume, grey matter density, and cortical thickness extracted from structural MRI through Free Surfer and voxel - based morphometry [13]. A variant in APOE (rs 429358) was associated with volumetric measures of the left and right amygdala and hippocampus regions, right middle temporal lobe, left and right inferior parietal lobe and right cerebral white matter [14]. This association was all significant at $\mathrm{p}<10-7$.

TOMM40 is a gene adjacent to APOE and is reported to be a contributor to LOAD risk, though this is detected 10 [15]. It encodes a channel - forming subunit of the multi subunit translocase of the outer mitochondrial membrane (TOMM complex), which place a role in the transport of cytoplasmic peptides and protein in the mitochondrial.

Given that mitochondrial dysfunction has been widely implicated in the etiology of LOAD, this association of TOMM 40 with LOAD was logical. TOMM40 has been associated with measures of brain structure volume, grey matter density and cortical thickness extracted from structural MRI: A variant in TOMM40 (rs2075650) was significantly associated with bilateral hippocampal volume and left amygdala volume [16]. In PET imaging GWAS, variant in TOMM40 displayed significant association which amyloid beta levels but, there association signals disappear $(\mathrm{P}>0.05)$ when APOE e3 status was included as a covariate, so the validity of the association TOMM40 with LOAD and it is endophenotype is still contentious 10 .

BCHE, located in chromosome 3, encodes butyryl cholinesterase and has been associated with amyloid beta plaques and neurofibrillary tangles along with amyloids angiopathy in AD [17]. Together variants in BCHE and APOE explained $15 \%$ of the variants in amyloid LOAD [18]. The risk variant of BCHE has been associated with further risk of LOAD in APOE e4 carriers and with cognitive declined in the later stage of the illness [19]. However, some studies has found that the risk variant of BCHE has important role in the progression of $\mathrm{AD}$ in which subjects carrying the risk allele, or $\mathrm{K}$-variant, exhibited is slower rate of cognitive declined $78 \%$. These observations have potential implications for the treatment of $\mathrm{AD}$ with cholinesterase inhibitors that inhibit the production of butyryl cholinesterase as the disease progresses.

GRIN2B, located on chromosomes 12, encodes the N-methyl$\mathrm{d}$-aspartate (NMDA) glutamate receptor NR2B subunit that has previously been associated with temporal lobe volume, excitotoxic cell disk and is already a therapeutic target in $\mathrm{AD}$ [20]. In this study a variant in GRIN2B (rs10845840) was associated with bilateral temporal lobe volume with $\mathrm{p}<5 \times 10-7$ and the risk alleles for lower temporal lobe volume at this SNP were significantly over represented in $\mathrm{AD}$ and $\mathrm{MCI}$ subjects versus controls [21].

An association with DHCR24, encoding 24-dehydroxycholesterol reductase, which confers resistance to amyloid, was discovered in the ADNI dataset using PiB-PET imaging as a quantitative trait [22]. The minor allele of an intronic SNP (rs7551288) within this gene was associated with lower amyloid beta load as measured by PiB uptake. Major allele homozygotes had higher amyloid beat load as measured by $\mathrm{PiB}$ uptake in frontal regions. Because of the role of DHCR24 in conferring resistance to amyloid beta and oxidative stress-induced apoptosis, the others posit that this result supports as neuroprotective role.

Using structural MRI derived quantitative traits derived from Caucasian subjects from ADNI, Shen, et. Al. reported genetic associations with SNPs located near EPHA4, TP63 and NXPH1 14, and another group has shown an association with EFNA5, CAND1, MAGI2, ARSB, and PRUNE2 [23]. These genes are involved in the regulation of protein degradation apoptosis, neuronal loss and neurodevelopment. Thus, they have been identified as candidate genes associated with an increased risk of developing AD.

Exploration beyond single gene association studies is necessary for complex diseases with complex etiologies and pathologies. This is just beginning to be explored, using quantitative traits derived from imaging modalities as the phenotype of interest. Previous genegene interaction studies using quantitative traits from brain images of patients with LOAD have implicated interactions between: CR1 and APOE, and GSK3B and amyloid genes using quantified amyloidbased PET as the outcome variable 21. And calcium signaling and axon guidance genes using quantified entorhinal and hippocampal volumes 23 .

Through PET has been approved for clinical detection of amyloid beta plaque pathology in vivo, since it involves radiation exposure, 
it is not ideal for routine screening or monitoring of patients, particularly before clinical symptoms are present. New biomarkers of amyloid beta pathology are needed and the exploration of the use of T1p MRI in humans in vivo is the next to validate its use as a biomarker of this pathology. If $\mathrm{T} 1 \mathrm{p}$ is validated as a proxy for amyloid beta deposition, it could easily be implemented to monitor diseases progress or classify patients in research and clinical trials. Thus, the first goal of this research is to analyze the utility of T1p MRI in human adults with DS, who have a high probability of amyloid beta deposition compared adults in the general population.

Further, we will explore the "accelerated brain aging" hypothesis in DS patients put forward by Bleacher, et.al. We will analyze region of interest volume using T1-weighted structural MR images in subjects with DS and compare age-related changes in these measurements to those in normal control subjects and also in subjects with another neurodevelopmental disorder (Williams Syndrome) to further explore the hypothesis. With the quantified T1- weighted images, we will extract meaningful endophenotypes to evaluate the relationship between both APOE status and dementia status as measured by neuropsychological tests and each of these endophenotypes.

The final goal of this research is to employ imaging genetics techniques in the large, publicly available ADNI dataset to tease apart the genetic etiology of the amyloid and neuro-atrophy pathologies of LOAD. As described above, the etiology of LOAD still eludes us despite its prevalence and considerable research efforts devoted to it. We will employ an innovative strategy to address two major challenges in LOAD research: (1) clinical heterogeneity and ((2) biological interactions by using PET and MRI derive quantitative endophenotypes to dissect clinical heterogeneity, and by directly investigating gene-gene interactions for their association with these endophenotypes.

\section{Acknowledgements}

None.

\section{Conflict of Interest}

No conflict of interest.

\section{References}

1. Donner A, Eliasziw M (1994) Statistical implications of the choice between a dichotomous or continuous trait in studies of interobserver agreement. Biometrics 50(2): 550-555.

2. Gottesman II, Gould T (2003) The Endophenotype Concept in Psychiatry: Etymology and Strategic Intentions. Am. J. Psychiatry 160(4): 636-645.

3. McKhann GM, Knopman DS, Chertkow H, Hyman BT, Jack CR, et al. (2011) The diagnosis of dementia due to Alzheimer's disease: recommendations from the national institute on Aging-Alzheimer's Association workgroups on diagnostic guidelines for Alzheimer's disease. Alzheimer's. Dement. 7(3): 263-269.

4. Potkin SG, Turner JA, Guffanti G, Lakatos A, Torri F, et al. (2009) Genome-wide strategies for discovering genetic influences on cognition and cognitive disorders: methodological considerations. Cogn. Neuriopsychiatry 14(4-5): 391-418.

5. Peper JS, Brouwer Rm, Boomsma DI, Khan RS, Hulshoff Pol HE (2007) Genetic influences on human brain structure: a review of brain imaging studies in twins. Hum Brain Mapp 28(6): 464-73.
6. Hinrichs AL, Mintun MA, Head D, Fagan AM, Holtzman DM, et al. (2010) Cortical Binding of Pittsburgh compound B, an endophenotype for genetic studies of Alzheimers disease. Biol Psychiatry 67(6): 581-583.

7. Eisenberg DTA, Kuzawa CW, Hayes MG (2010) Worldwide ellele frequencies of the human apoliprotein E. gene: climate, Local adaptations, and evolutionary history. Am. J. Phys. Anthropol. 143(1): 100-111.

8. Qiu C Kivipelto M, Agüero Torres H, Winblad B, Fratiglioni L (2004) Risk and protective effects of the APOE gene towards Alzheimer's disease in the Kungsholmen project: variation by age and sex. J. Neurol. Neurosurg. Psychiatry 75(6): 828-833.

9. Tang MX, Stern Y, Marder K, Bell K, Gurland B, et al. (1998) The APOEepsilon4 allele and the risk of Alzheimer's disease among African Americans, whites and Hispanics. JAMA 279(10): 751-755.

10. Ramanan VK, Risacher SL, Nho K, Kim S, Swaminathan S, et al. (2013) APOE and BCHE as modulators of cerebral amyloid deposition: a florbetapir PET genome-wide association study. Mol Psychiatry 19(3): 351-357.

11. Reiman EM, Chen K, Liu X, Bandy D, Yu M, et al. (2009) Fibrillar amyloidbeta burden in cognitively normal people at 3 levels of genetic risk for Alzheimers disease. Proc Natl Acad Sci USA 106(16): 6820-6825.

12. Kemppainen NM, Aalto S, Wilson IA, Någren K, Helin S, et al. (2007) PET amyloid ligand [11C] PIB uptake is increased in mild cognitive impairment. Neurology 68(19): 1603-1606.

13. Okello A, Koivunen J, Edison P, Archer HA, Turkheimer FE, Någren K, et al. (2009) Conversion of amyloid positive and negative MCI to AD over 3 years: an 11C-PIB PET study. Neurology 73(10): 754-760.

14. Shen L, Kim S, Risacher SL, Nho K, Swaminathan S, et al. (2010) Whole genome association study of brain-wide imaging phenotypes for identifying quantitative trait loci in MCI and AD: A study of the ADNI cohort. Neuroimage 53(3): 1051-1063.

15. Potkin SG, Guffanti G, Lakatos A, Turner JA, Kruggel F, et al. (2009) Hippocampal atrophy as a quantitative trait in a genome-wide association study identifying novel susceptibility genes for Alzheimer's disease. 4(8): e6501.

16. Darvesh S, Hopkins DA, Geula C (2003) Neurobiology of butyrylchilinesterase. Nat Rev Neurosci 4(2): 131-138.

17. Lehmann DJ, Johnston C, Smith AD (1997) Synergy between the genes for butyrylchilinesterase $\mathrm{K}$ variant and apolipoprotein $\mathrm{E} 4$ in late-onset confirmed Alzheimer's disease. Hum Mol Genet 6(11): 1933-1936.

18. Holmes C, Ballard C, Lehmann D, David Smith A, Beaumont H, et al. (2005) Rate of progression of cognitive decline in Alzheimer's disease: effect of butyrylchilinesterase K gene variation. J. Neurol. Neurosurg. Psychiatry 76(5): 640-643.

19. Stein JL, Hua X, Morra JH, Lee S, Hibar DP, et al. (2010) Genome wide analysis reveals novel genes influencing temporal love structural with relevance to neurodegeneration in Alzheimer's disease. Neuroimage 51(2): 542-554.

20. Swaminathan S, Shen L, Risacher SL, Yoder KK, West JD, et al. (2012) Amyloid pathway-based candidate gene analysis of [(11)C] PiB-PET in the Alzheimers disease Neuroimaging Initiative (ADNI) cohort. Brain Imaging Behav. 6(1): 1-15.

21. Thambisetty M, An Y, Nalls M, Sojkova J, Swaminathan S, et al. (2013) Effect of Complement CR1 on Brain Amyloid Burden during Aging and its Modification by APOE Genotype. Biol. Psychiatry 73(5): 422-428.

22. Hohman TJ, Koran MEI, Thornton Wells TA (2013) Interactions between GSK3B and amyloid genes explain variance in amyloid burden. Neurobiol Aging 35(3): 460-465.

23. Beacher F, Daly E, Simmons A, Prasher V, Morris R, et al. (2010) Brain anatomy and aging in non-demented adults with Down 's syndrome: an in vivo MRI study. Psychol Med 40(4): 611-619. 